\title{
A commentary on de novo MEIS2 mutation causes syndromic developmental delay with persistent gastro-esophageal reflux
}

\author{
Rie Takai and Tohru Ohta \\ Journal of Human Genetics (2016) 61, 773-774; doi:10.1038/jhg.2016.81; published online 7 July 2016
}

$\mathrm{H}$ omeobox genes encode transcription factors that regulate the body plan, especially for segmental patterning during early embryonic development. In humans, $>230$ homeobox genes have been isolated. ${ }^{1}$ These genes encode homeodomain proteins that interact directly with the regulatory regions of target genes. The homeodomain consists of a helix-turn-helix structure with 60 amino acids. Some homeobox gene groups have an additional three-amino-acid-loop extension (TALE) between a-helices 1 and 2 in the homeodomain, making up the so-called TALE superfamily ${ }^{2}$ of which $P B X$, $R B C$ and MEIS/PREP are members. The homeodomain in TALE superfamily proteins binds to the target DNA region in the same way as major homeobox proteins do, with the cooperation of other Hox genes (Figure 1a).

The Meisl locus was initially isolated as a common site of viral integration involved in myeloid leukemia in mice. Meis2 was isolated as a highly similar gene but not identical to Meis $1 .^{3}$ Meis family products constitute a dimeric or trimeric complex with other homeodomain proteins (Figure 1a), ${ }^{2}$ and their combination patterns enhance the specificity of DNA binding.

Similar to most other TALE family genes, MEIS1 and MEIS2 are highly expressed in neuroblastoma cell lines. ${ }^{4}$ MEIS2 has been reported to be a critical transcription factor involved in limb development, hindbrain patterning, lens morphogenesis, retinal differentiation, heart tube formation, normal mesencephalic development in animal

R Takai and T Ohta are at The Research Institute of Personalized Health Sciences, Health Sciences University of Hokkaido, Hokkaido, Japan

E-mail: ohta@hoku-iryo-u.ac.jp model $s^{5}$ and ganglionic eminence in the human forebrain. ${ }^{6}$

Meis2 is expressed in neural crest cells of mice, and according to Machon et al., ${ }^{7}$ Meis2-deficient embryos display an abnormal heart outflow tract with persistent truncus arteriosus, impaired trigeminal $\mathrm{V}$ and cranial nerves VII and VIII, thinner corneas and undeveloped eyelids. The authors also showed that the conditional inactivation of Meis2 manifested in perturbed development of the craniofacial skeleton, with severe anomalies in cranial bones and cartilages, as well as

a

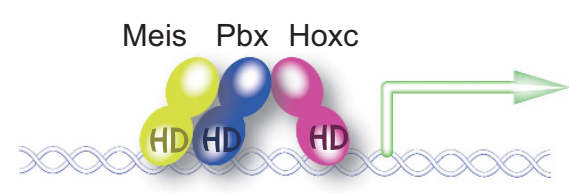

abnormalities in the heart and cranial nerve. ${ }^{7}$ Thus, the human MEIS2 gene may also contribute to tissue development originated from the neural crest.

Recently, four papers described genomic deletions involving MEIS2 and its point mutations. ${ }^{5,8-10}$ Crowley et al. ${ }^{8}$ reported a case of cleft soft palate, ventricular septal defect and bilateral moderate hearing loss in association with a deletion of MEIS2 exon 9 that had led to its haploinsufficiency; it was the smallest deletion among nine patients previously reported with haploinsufficiency

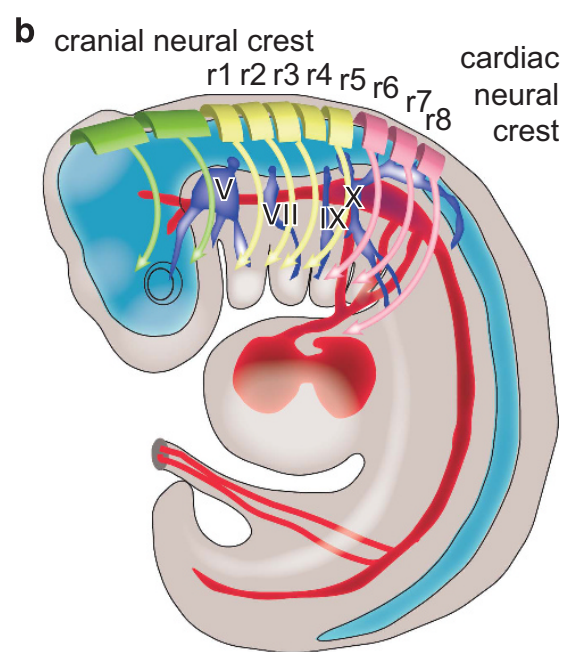

Figure 1 (a) The Meis protein works as a transcription factor. The schema is a putative example of the DNA-binding proteins Meis, $\mathrm{Pbx}$ and Hoxc, constituting a trimeric complex. ${ }^{2}$ The specificity was enhanced with the three homeodomains binding to target regulatory regions to activate a gene (arrow). (b) Migration of the neural crest in the embryo. The cranial neural crest at the anterior and posterior mid-brain migrates to a large part of the head comprising the face and skull. ${ }^{13}$ The hindbrain is segmented as rhombomeres ( $r 1-r 8$ ). The cardiac neural crest arising from rhombomeres $6-8$ migrates to and enters pharyngeal arches 3, 4 and 6 to become thyroid, parathyroid and thymus glands, and also generates the endothelium of aortic arch arteries and the septum between the aorta and the pulmonary artery. ${ }^{13}$ The sensory neurons of cranial nerve ganglia V, VII, IX and X are also neural crest-derived, forming with cells from the epibranchial placode. 
of the gene. Johansson et al. ${ }^{5}$ summarized nine patients with MEIS2 abnormality, among whom seven had cleft palate, four suffered from atrial or ventricular septal defect, and eight had mild to severe intellectual disability. Very recently, Louw et al. ${ }^{9}$ reported a patient with severe phenotypes, including cleft palate and congenital heart defect, who carried a one-amino-acid deletion mutation (p.Arg333del) in the MEIS2 Hox domain. This amino acid deletion likely caused a disturbance of the protein's DNA-binding competence. Further evidence of a point mutation in MEIS2 is reported in the current issue of the Journal of Human Genetics, where Fujita et al. ${ }^{10}$ described a patient with a nonsense mutation $\left(\mathrm{p}\right.$. Ser204*) at the $5^{\prime}$-region of the gene. This stop-gain mutation may have produced a truncated protein that had lost the critical Hox domain of MEIS2. ${ }^{10}$ The patient showed severe intellectual disability, severe motor/ verbal developmental delay, cleft palate, cardiac septal defect, hypermetropia, severe feeding difficulties with gastro-esophageal reflux and constipation. It was clearly demonstrated that the cleft palate/lip and cardiac malformation were caused by a nonsense mutation in MEIS2. This nonsense mutation might be a similar condition to haploinsufficiency from MEIS1 deletion, or the truncated protein may have more severe clinical features than its haploinsufficiency (see the table in this issue $^{10}$ ) through possible dominant-negative effects. To elucidate these possibilities, further experiments will be required.

Neural crest cells are multipotent embryonic cells derived from the ectoderm transiently located between the neural tube and epidermis (Figure 1b). If these multipotent cells are deficient in the neural crest, an abnormal heart outflow associated with persistent truncus arteriosus and abnormal cranial nerves could occur. The neural crest is anatomically divided into four regions: the cranial neural crest, cardiac neural crest, trunk neural crest, and vagal and sacral neural crest. The cranial neural crest can produce a large part of the head, including the bones, cartilages, connective tissues, pigment cells, glial cells and peripheral neurons. The sensory neuron of the vagus nerve also originates from the cranial neural crest with the combination of epibranchial placodes. ${ }^{11}$ The parasympathetic innervation by the vagus nerve spreads widely to the stomach, small intestine and proximal colon. The afferent vagus, especially the 5-HT3 receptor on neuronal cells, modulates several physiological and pathophysiological conditions, including vagal reflexes, nausea and vomiting. ${ }^{12}$ Inadequate migration from the neural crest and abnormal development of the vagus nerve, caused by MEIS2 abnormality, must be the reason for the feeding difficulty associated with gastro-esophageal reflux observed in the patient described by Fujita et al. ${ }^{10}$

In conclusion, the patient described here with a nonsense mutation, ${ }^{10}$ the patient with an exon 9 deletion and the patient with a one-amino-acid deletion at the homeodomain may all suggest a new clinical syndrome caused by MEIS2 abnormalities with phenotypes of cleft palate/lip, cardiac anomaly and feeding difficulty with gastro-esophageal reflux.

\section{CONFLICT OF INTEREST}

The authors declare no conflict of interest.
1 Holland, P. W., Booth, H. A. \& Bruford, E. A. Classification and nomenclature of all human homeobox genes. BMC Biol. 5, 47 (2007).

2 Merabet, S. \& Mann, R. S. To be specific or not: the critical relationship between Hox and TALE proteins. Trends Genet. 6, 334-347 (2016).

3 Nakamura, T., Jenkins, N. A. \& Copeland, N. G. Identification of a new family of Pbx-related homeobox genes. Oncogene 13, 2235-2242 (1996).

4 Geerts, D., Schilderink, N., Jorritsma, G. \& Versteeg, R. The role of the MEIS homeobox genes in neuroblastoma. Cancer Lett. 197, 87-92 (2003).

5 Johansson, S., Berland, S., Gradek, G. A., Bongers, E., de Leeuw, N., Pfundt, R et al. Haploinsufficiency of MEIS2 is associated with orofacial clefting and learning disability. Am. J. Med. Genet. A 164 A, 1622-1626 (2014).

6 Larsen, K. B., Lutterodt, M. C., Laursen, H., Graem, N., Pakkenberg, B., Møllgård, K. et al. Spatiotemporal distribution of PAX6 and MEIS2 expression and total cell numbers in the ganglionic eminence in the early developing human forebrain. Dev. Neurosci. 32, 149-162 (2010).

7 Machon, O., Masek, J., Machonova, O., Krauss, S. \& Kozmik, Z. Meis2 is essential for cranial and cardiac neural crest development. BMC Dev. Biol. 6, 40 (2015).

8 Crowley, M. A., Conlin, L. K., Zackai, E. H., Deardorff, M. A., Thiel, B. D. \& Spinner, N. B. Further evidence for the possible role of MEIS2 in the development of cleft palate and cardiac septum. Am. J. Med. Genet. A 152 A, 1326-1327 (2010).

9 Louw, J. J., Corveleyn, A., Jia, Y., Hens, G., Gewillig, M. \& Devriendt, K. MEIS2 involvement in cardiac development, cleft palate, and intellectual disability. Am. J. Med. Genet. A 167 A, 1142-1146 (2015).

10 Fujita., A., Isidor, B., Piloquet, H., Corre, P., Okamoto, N., Nakashima, $\mathrm{M}$ et al. De novo MEIS2 mutation causes syndromic developmental delay with persistent gastro-esophageal reflux. J. Hum. Genet 61, 835-838 (2016).

11 Thompson, H., Blentic, A., Watson, S., Begbie, J. \& Graham, A. The formation of the superior and jugular ganglia: insights into the generation of sensory neurons by the neural crest. Dev. Dyn. 239, 439-445 (2010).

12 Browning, K.N. Role of central vagal 5-HT3 receptors in gastrointestinal physiology and pathophysiology. Front Neurosci. 9, 413 (2015).

13 Gilbert, S. F. Developmental Biology, 10th edn (Sinauer Associates, Inc, Sunderland, MA, USA, 2014). 